Research Paper

\title{
Impact of Age at Diagnosis on Outcomes in Men with Castrate-Resistant Prostate Cancer (CRPC)
}

\author{
Michael R Humphreys ${ }^{1}$, Kimberly A Fernandes ${ }^{2}$, Srikala S Sridhar ${ }^{3}$ \\ 1. Division of Medical Oncology, British Columbia Cancer Agency, Vernon, BC, Canada; \\ 2. Department of Biostatistics, Princess Margaret Hospital, University Health Network, Toronto, ON, Canada; \\ 3. Department of Medical Oncology and Hematology, Princess Margaret Hospital, University Health Network, Toronto, ON, Canada.
}

$\triangle$ Corresponding author: Srikala S. Sridhar MD MSc FRCPC, Medical Oncologist, Princess Margaret Hospital, Assistant Professor, University of Toronto. 5-222, 610 University Avenue, Toronto, ON, Canada M5G 2M9, TEL: 416-946-4501 2662 FAX: 416-946-6546. Srikala.Sridhar@uhn.ca.

(C) Ivyspring International Publisher. This is an open-access article distributed under the terms of the Creative Commons License (http://creativecommons.org/ licenses/by-nc-nd/3.0/). Reproduction is permitted for personal, noncommercial use, provided that the article is in whole, unmodified, and properly cited.

Received: 2013.01.29; Accepted: 2013.03.01; Published: 2013.03.21

\begin{abstract}
Background: The association between age and outcomes in men with castrate resistant prostate cancer (CRPC) is not well understood.

Objective: We aimed to evaluate CRPC patients to determine if their age at initial diagnosis impacted their cancer specific outcomes.

Design, Setting, and Participants: A retrospective chart review was conducted on 333 consecutive CRPC patients treated at the Princess Margaret Hospital (PMH) between 1995 and 2005. Patients were divided into 4 age categories, (A) <55, (B) 55-64, (C) 65-74 (reference), and (D) $\geq 75$ years (yrs).

Outcome Measurements and Statistical Analysis: Primary endpoints included impact of age at diagnosis on overall survival (OS) and on prostate cancer specific survival. Secondary endpoints were time from diagnosis to development of CRPC, time from CRPC to death, and time from diagnosis to bone metastases.

Results and Limitations: The median OS from diagnosis to death was: Group A 5.5 yrs $(95 \% \mathrm{Cl}$ 3.0-7.5); Group B 6.7 yrs (95\% Cl 5.9-8.4); Group C 7.8 yrs (95\% Cl 6.6-9.3); and Group D 4.3 years $(95 \% \mathrm{Cl}$ 2.9-5.0). The hazard ratio (HR) for death in Group D was 2.58 (95\% Cl I.58-4.2I, $\mathrm{p}=0.0002)$; and in Group A was I.49 (95\% Cl 0.90-2.46, $\mathrm{p}=0.13)$. The duration of hormone sensitivity in Group D was less and predictive of OS, as was Gleason Score $\geq 8$ and Stage 4 disease at diagnosis.

Conclusions: Age at initial diagnosis appears to impact on outcome of patients who subsequently develop CRPC with a bimodal distribution of risk, with the shortest survivals in the $\geq 75$ and $<55$ groups.
\end{abstract}

Key words: Castrate-resistant prostate cancer, overall survival, age at diagnosis, hormone-refractory.

\section{Introduction}

In 2012, an estimated 241,740 men were diagnosed with prostate cancer in the US, of which approximately 28, 170 died from the disease. ${ }^{1}$ While a diagnosis of prostate cancer in men under the age of 50 is rare, the incidence and mortality increases steadily with age. Several disease-specific factors (e.g. 
stage, tumor grade, prostate-specific antigen (PSA) level) and patient-specific factors (e.g. co-morbidity, primary treatment modality, and functional status) have been identified as important predictors of survival. ${ }^{2-6}$ However, the influence of age on outcomes in prostate cancer remains controversial. 2,6-9

In a review of 34 studies involving patients with localized prostate cancer, six studies found that young age at diagnosis carried a poor prognosis; five studies showed young age portended a good prognosis, and the remaining 23 studies found no association. ${ }^{9}$ A more contemporary study analyzing 4003 patients on androgen deprivation therapy (ADT) enrolled in the Cancer of the Prostate Strategic Urological Research Endeavor (CaPSURE) study from 1995-2007 found men $<65$ at diagnosis were at a significantly higher risk of developing metastasis $(\mathrm{HR}=2.11) \cdot{ }^{10}$ Supporting this observation was a Surveillance, Epidemiology, and End Results (SEER) database study showing that patients diagnosed at a younger age had worse survival, especially when accompanied by advanced histological grade and stage. ${ }^{11}$ In contrast, a second SEER based study did not show an impact of age on survival; however, this study also failed to stratify patients into multiple age categories. ${ }^{12}$

For CRPC patients, Gleason score, PSA, PSA doubling time, hemoglobin (Hgb), lactate dehydrogenase (LDH), alkaline phosphatase (ALP), performance status (PS), pain at baseline, chemotherapy type, ethnicity, presence of visceral disease and advanced age have all been identified as factors that impact OS. ${ }^{13-15}$ Only one study, by Halabi et al, has directly assessed the impact of age on outcome in CRPC. ${ }^{14}$ This study, which utilized pooled data from 8 CALGB trials, found that men aged 80-89 were at increased risk for death, compared to their younger counterparts. Interestingly, they also reported that younger men (age 50-59) were at a $25 \%$ increased risk of clinical progression and $26 \%$ increased risk of prostate cancer death compared to those aged 70-79 but this did not translate into a decreased OS $(\mathrm{p}=0.172) .{ }^{14}$

Understanding the relationship between age and outcomes may not only have prognostic implications, but in the era of molecular targeted therapy and personalized medicine may also have important therapeutic implications. In our study, we evaluated 333 consecutive CRPC patients to determine if age at initial diagnosis of prostate cancer impacted on OS.

\section{Patients and Methods}

Following ethics approval, a retrospective chart review was performed on 3295 consecutive prostate cancer patients referred to the PMH between January
1, 1995 and December 31, 2005. PMH registry database and chart review identified 333 patients that fulfilled inclusion criteria. Vital statistics were obtained by linking to the Cancer Care Ontario database (accessed July 31, 2012).

Eligible patients had histologically confirmed adenocarcinoma of the prostate with disease progression after androgen ablation as indicated by two consecutive PSA rises or with measurable disease progression as defined by RECIST criteria. ${ }^{16}$ Summary statistics for demographic and clinical factors were generated for each case at diagnosis and during treatments. The Charlson Comorbidity Index (CCI) was used as an assessment of comorbidities at the time of diagnosis. ${ }^{77,18}$

The primary endpoints were the impact of age at diagnosis on OS and on prostate cancer specific survival. Secondary endpoints included impact of age on a) time from diagnosis to development of CRPC $b$ ) time from CRPC to death and c) time from diagnosis to bone metastases. A prespecified subset analysis evaluated the impact of age on OS, progression free survival (PFS) and PSA-PFS in patients receiving chemotherapy. PFS was defined as a composite of PSA progression, bone progression, nodal or visceral progression, and death, while PSA-PFS was defined by $\mathrm{PCWG}_{2}$ criteria. $^{19}$

OS curves were created by the Kaplan-Meier approach. Univariate and multivariate Cox $\mathrm{PH}$ regression analyses were conducted. All covariates were entered into the multivariate model. All statistical analyses were performed using SAS version 9.1 and $\mathrm{R}$ version 2.7.1. All statistical tests were two-sided and $p$-values $\leq 0.05$ were considered statistically significant. Multiple imputation methodology was performed when indicated for incomplete data sets. Patients lost to follow-up were censored at last known contact. In order to account for an uneven distribution of patients across the 4 age groups we performed our sample size calculation post-hoc based on the smallest group. The total sample size required to detect the increased risk of death of $32 \%$ on multivariate analysis $(\mathrm{HR}=1.32)$ identified in this study, at a power of $80 \%$ with a 2 -sided $\mathrm{p}=0.05$ was 293 patients.

\section{Results}

\section{Baseline Characteristics}

Amongst the 333 eligible CRPC patients, average age at diagnosis was 65; most patients had Gleason scores of 8-10; median PSA was 146, LDH 223, ALP 144 and $\mathrm{Hgb} 120$. A greater proportion of patients in Groups A and D presented with Stage 4 disease. Group D patients had more comorbidities and were 
less likely to have undergone definitive local therapy. ation of chemotherapy, Group D patients had poorer However, they achieved a quicker PSA nadir on ADT at an average of only 9.2 months (mos). Prior to initiPS and were less likely to receive chemotherapy than younger patients (Table 1).

Table I. Baseline characteristics of study population $(n=333)$.

\begin{tabular}{|c|c|c|c|c|c|}
\hline Age at Diagnosis (years) & Group A $<55$ & Group B $55-64$ & Group C 65 - 74 & Group D $\geq 75$ & Total \\
\hline$n(\%)$ & $30(9 \%)$ & $124(37 \%)$ & $145(43 \%)$ & $35(10 \%)$ & $333(100 \%)$ \\
\hline \multicolumn{6}{|l|}{ Characteristics at Time of Diagnosis } \\
\hline \multicolumn{6}{|l|}{ Stage at Dx† } \\
\hline $1-2$ & $4(14 \%)$ & $19(17 \%)$ & $40(31 \%)$ & $7(21 \%)$ & $70(23 \%)$ \\
\hline 3 & $5(17 \%)$ & $38(34 \%)$ & $35(27 \%)$ & $8(24 \%)$ & $86(29 \%)$ \\
\hline 4 & $20(69 \%)$ & $54(49 \%)$ & $53(41 \%)$ & $18(55 \%)$ & $145(48 \%)$ \\
\hline \multicolumn{6}{|l|}{ PSA at Dx } \\
\hline $0-<10$ & $10(40 \%)$ & $23(23 \%)$ & $31(28 \%)$ & $6(18 \%)$ & $70(26 \%)$ \\
\hline $10-<20$ & $2(8 \%)$ & $22(22 \%)$ & $24(21 \%)$ & $2(6 \%)$ & $50(18 \%)$ \\
\hline $20-<100$ & $7(28 \%)$ & $34(34 \%)$ & $33(34 \%)$ & $11(33 \%)$ & $90(33 \%)$ \\
\hline$\geq 100$ & $6(24 \%)$ & $22(22 \%)$ & $19(17 \%)$ & $14(42 \%)$ & $61(23 \%)$ \\
\hline \multicolumn{6}{|l|}{ Prostatectomy at Dx† } \\
\hline No & $24(80 \%)$ & $94(77 \%)$ & $120(83 \%)$ & $34(97 \%)$ & $272(82 \%)$ \\
\hline Yes & $6(20 \%)$ & $28(23 \%)$ & $24(17 \%)$ & $1(3 \%)$ & $59(18 \%)$ \\
\hline \multicolumn{6}{|l|}{ RT at Dx } \\
\hline No & $19(63 \%)$ & $80(66 \%)$ & $89(62 \%)$ & $28(80 \%)$ & $216(65 \%)$ \\
\hline Yes & $11(37 \%)$ & $42(35 \%)$ & $55(38 \%)$ & $7(20 \%)$ & $115(35 \%)$ \\
\hline \multicolumn{6}{|l|}{ Gleason at Dx } \\
\hline$<=6$ & $2(7 \%)$ & $6(6 \%)$ & $14(11 \%)$ & $3(11 \%)$ & $25(9 \%)$ \\
\hline 7 & $8(30 \%)$ & $34(33 \%)$ & $40(33 \%)$ & $5(18 \%)$ & $87(31 \%)$ \\
\hline $8-10$ & $17(63 \%)$ & $63(61 \%)$ & $68(56 \%)$ & $20(71 \%)$ & $168(60 \%)$ \\
\hline \multicolumn{6}{|l|}{ CCI at time of Dx† } \\
\hline 0 & $22(73 \%)$ & $59(50 \%)$ & $56(40 \%)$ & $8(25 \%)$ & $145(45 \%)$ \\
\hline $1-2$ & $7(23 \%)$ & $46(39 \%)$ & $66(47 \%)$ & $16(50 \%)$ & $135(42 \%)$ \\
\hline $3-4$ & $1(3 \%)$ & $9(8 \%)$ & $14(10 \%)$ & $8(25 \%)$ & $32(10 \%)$ \\
\hline$>4$ & $0(0 \%)$ & $4(3 \%)$ & $3(2 \%)$ & $0(0 \%)$ & $7(2 \%)$ \\
\hline \multicolumn{6}{|c|}{ Time in Years to Bone Mets or Death from Dx } \\
\hline (median) $\dagger$ & 1.15 & 3.95 & 4.07 & 2.08 & 3.44 \\
\hline \multicolumn{6}{|l|}{ Characteristics at Time of CRPC } \\
\hline \multicolumn{6}{|l|}{ PSA Nadir on ADT $\dagger$} \\
\hline$<4$ & $19(63 \%)$ & $85(79 \%)$ & $92(75 \%)$ & $18(56 \%)$ & $214(73 \%)$ \\
\hline$\geq 4$ & $11(37 \%)$ & $23(21 \%)$ & $31(25 \%)$ & $14(44 \%)$ & $79(27 \%)$ \\
\hline $\begin{array}{l}\text { Time in Months to PSA Nadir fro } \\
\text { Initiation (mean) } \dagger\end{array}$ & 22.5 & 19.6 & 21.5 & 9.21 & 20.0 \\
\hline \multicolumn{6}{|l|}{ Visceral Disease } \\
\hline No & $21(70 \%)$ & $101(82 \%)$ & $125(86 \%)$ & $30(86 \%)$ & $277(83 \%)$ \\
\hline Yes & $9(30 \%)$ & $22(18 \%)$ & $20(14 \%)$ & $5(14 \%)$ & $56(17 \%)$ \\
\hline \multicolumn{6}{|l|}{ Antiandrogen Withdrawal Response } \\
\hline No & $19(63 \%)$ & $85(79 \%)$ & $92(75 \%)$ & $18(56 \%)$ & $195(74 \%)$ \\
\hline Yes & $11(37 \%)$ & $23(21 \%)$ & $31(25 \%)$ & $14(44 \%)$ & $68(26 \%)$ \\
\hline \multicolumn{6}{|c|}{ Characeristics at Time of Chemotherapy Consideration } \\
\hline \multicolumn{6}{|l|}{ ECOG Performance Status $\dagger$} \\
\hline $0-1$ & $27(96 \%)$ & $83(72 \%)$ & $91(69 \%)$ & $16(53 \%)$ & $217(71 \%)$ \\
\hline 2 & $1(4 \%)$ & $26(22 \%)$ & $29(22 \%)$ & $3(10 \%)$ & $59(19 \%)$ \\
\hline $3-4$ & $0(0 \%)$ & $7(6 \%)$ & $12(9 \%)$ & $11(37 \%)$ & $30(10 \%)$ \\
\hline \multicolumn{6}{|l|}{ PSA Doubling Time $†$} \\
\hline $0-30$ & $9(32 \%)$ & $30(26 \%)$ & $28(21 \%)$ & $3(9 \%)$ & $70(23 \%)$ \\
\hline$\geq 30-60$ & $6(21 \%)$ & $46(40 \%)$ & $38(29 \%)$ & $13(38 \%)$ & $103(33 \%)$ \\
\hline$\geq 60$ & $13(46 \%)$ & $39(34 \%)$ & $67(50 \%)$ & $18(53 \%)$ & $137(44 \%)$ \\
\hline Median PSA (mg/dL) & 113 & 158 & 131 & 154 & 146 \\
\hline
\end{tabular}




\begin{tabular}{|c|c|c|c|c|c|}
\hline Median LDH (IU/L) & 236 & 244 & 223 & 208 & 223 \\
\hline Median ALP (IU/L) & 161 & 149 & 144 & 123 & 144 \\
\hline Median Hgb (g/L) & 124 & 120 & 120 & 115 & 120 \\
\hline \multicolumn{6}{|l|}{ Chemotherapy $\dagger$} \\
\hline Docetaxel & $11(37 \%)$ & $30(26 \%)$ & $29(20 \%)$ & $5(15 \%)$ & $75(23 \%)$ \\
\hline Mitoxantrone & $6(20 \%)$ & $27(23 \%)$ & $47(33 \%)$ & $12(35 \%)$ & $92(29 \%)$ \\
\hline Docetaxel and Mitoxantrone & $8(27 \%)$ & $37(32 \%)$ & $29(20 \%)$ & $0(0 \%)$ & $74(23 \%)$ \\
\hline None & $5(17 \%)$ & $20(17 \%)$ & $37(26 \%)$ & $17(50 \%)$ & $79(25 \%)$ \\
\hline Other & $0(0 \%)$ & $2(2 \%)$ & $0(0 \%)$ & $0(0 \%)$ & $2(<1 \%)$ \\
\hline
\end{tabular}

Abbreviations: PSA, prostate specific antigen; Dx, diagnosis; RT, radiotherapy; CCI, Charleston comorbidity index, CRPC, castration resistant prostate cancer; ADT, androgen deprivation therapy; ECOG, Eastern Cooperative Oncology Group; LDH, lactose dehydrogenase; ALP, alkaline phosphatase; Hgb, hemoglobin $(\dagger$, meets $\mathrm{p}<0.05$ statistical significance per Fisher's Exact Test or Chi-squared test).

\section{Time from Initial Diagnosis to Death}

After a median follow-up of 6.6 yrs, 255 of 333 patients $(77 \%)$ had died. Median survival in Group A was 5.5 yrs; Group B was 6.7 yrs; Group C was 7.8 yrs; and Group D was 4.3 yrs with the differences between the groups being statistically significant (log-rank $\mathrm{p}<0.0001$, Table 2a, Fig 1). For the entire cohort, overall 5 -yr survival rate from diagnosis was $62 \%$, but 10 -yr survival rate was only $28 \%$. As shown in Table $2 \mathrm{~b}$, on univariate analysis there was a statistically significant increased risk of death associated with age $\geq 75$, HR of 2.58 ( $p=0.0002)$. Patients $<55$ also showed a trend towards an increased risk of death with a HR of 1.49 ( $p=0.13$ ). On multivariate analysis, age $\geq 75$ was associated with the largest HR for death (2.84, $\mathrm{p}<0.0001)$, followed by stage 4 disease $(\mathrm{HR}=2.83$, $\mathrm{p}<0.0001)$, and Gleason $\geq 8(\mathrm{HR}=2.57, \mathrm{p}=0.008$; Table $2 b)$.To account for non-prostate cancer deaths, which could be a source of bias, the above analyses were repeated for disease specific survival. The results remained similar to the OS analysis with HR of 1.7 $(p=0.04)$ for Group D and $1.5(p=0.09)$ for Group A on univariate analysis. On multivariate analysis the HR was preserved in the older cohort but the statistical significance was lost $(\mathrm{HR}=1.6 ; \mathrm{p}=0.16)$. Multivariate analysis suggested that stage at diagnosis was the primary driver $(\mathrm{HR}=2.2, \mathrm{p}=0.004)$ for increased risk of death. As noted above, patients at both extremes of age had an increased proportion of advanced stage disease at diagnosis.

When time from diagnosis to the development of bone metastasis and duration of hormone sensitivity were included in the analysis of OS, both were independently predictive of OS from diagnosis $(\mathrm{HR}=0.71$ per $1 \mathrm{yr}$ increase, $\mathrm{p}<0.001$, and $\mathrm{HR}=0.80$ per $1 \mathrm{yr}$ increase, $\mathrm{p}<0.001$, respectively). The time from diagnosis to death was not significantly influenced by CCI. Non-docetaxel based chemotherapy was associated with a worse prognosis compared to no therapy
$(\mathrm{HR}=2.01, \mathrm{p}=0.002)$. Time from initial diagnosis to death was divided into two clinically relevant time frames: a) time from initial diagnosis to CRPC and b) time from CRPC to death, and analyzed independently.

\section{a)Time from Diagnosis to CRPC}

Time from initial diagnosis to CRPC was $2.1 \mathrm{yrs}$ (Group A), 2.8 yrs (Group B), 3.4 yrs (Group C), and 1.3 yrs (Group D) (log-rank p<0.0001, Table 3, Fig 2). Multivariate analysis again yielded the largest HR for age $\geq 75$ (HR 2.64, p=0.0001), followed by Gleason score $\geq 8$ (HR 2.55, $\mathrm{p}=0.004$ ), and stage 4 disease at presentation $(\mathrm{HR}=2.21, \mathrm{p}<0.0001)$.

\section{b) Time from CRPC to death}

At the time of data-lock, 77\% (230/300) deaths were observed. The median time from CRPC to death was 2.9 yrs. There was no statistically significant difference in OS between the age stratified cohorts (Fig 3 ). The key factors that were independently predictive of adverse survival from CRPC to death were presence of visceral metastasis, absolute PSA nadir on ADT ( $>4 \mathrm{vs} \leq 4 \mathrm{ng} / \mathrm{ml})$, time to PSA nadir on ADT ( $<6$ vs $\geq 6 \mathrm{mos}$ ), and duration of hormone sensitivity determined by time from starting a gonadotropin releasing hormone $(\mathrm{GnRH})$ agonist to CRPC ( $<12 \mathrm{vs} \geq 12$ mos, Table 4). Anti-androgen withdrawal response occurred in $26 \%$ of patients and did not impact OS. There was no discernible difference between patients receiving docetaxel compared to no chemotherapy, but CRPC patients receiving non-docetaxel chemotherapy exhibited a reduced survival $(\mathrm{HR}=2.00$, $\mathrm{p}<0.0001)$. Variables measured at diagnosis did not influence survival from CRPC. We also found that age $<55$ at the time of CRPC diagnosis was associated with a trend to increased risk of death on univariate analysis $(\mathrm{HR}=1.78, \mathrm{p}=0.13)$ but not on multivariate analysis $(\mathrm{HR}=1.19, \mathrm{p}=0.62$, Fig 4$)$. 
Table 2a. Overall Survival (From Initial Diagnosis to Death).

\begin{tabular}{lllll}
\hline Age $(\mathrm{y})$ & Group A $<55$ & Group B 55 - 64 & Group C 65 - 74 & Group D $\geq 75$ \\
\hline Med. Survival (years) (95\% CI†) & $5.5(3.0-7.5)$ & $6.7(5.9-8.4)$ & $7.8(6.6-9.3)$ & $4.3(2.9-5.0)$ \\
$5 y$ Survival (\%) (95\% CI) & $52(33-68)$ & $67(58-75)$ & $66(58-73)$ & $35(19-51)$ \\
10y Survival (\%) (95\% CI) & $27(13-44)$ & $27(19-36)$ & $33(25-42)$ & $10(2-25)$ \\
\hline Abbreviations: $y$ years:Med, median: CRPC castration resistant prostate cancer; (t depicts log rank test statistical significance p<0.0001).
\end{tabular}

Abbreviations: $y$, years;Med, median; $C R P C$, castration resistant prostate cancer; ( $\dagger$ depicts log rank test statistical significance $p<0.0001)$.

Table 2b. Univariable and Multivariable Cox-PH Regression Analysis of Overall Survival.

\begin{tabular}{|c|c|c|c|c|}
\hline & Univariable & & Multivariable & \\
\hline & HR $(95 \%$ CI) & $p$-value & HR $(95 \%$ CI) & $p$-value \\
\hline Age $(y)$ & & 0.0009 & & 0.0004 \\
\hline Group A $<55$ & $1.49(0.90-2.46)$ & 0.13 & $1.32(0.77-2.27)$ & 0.32 \\
\hline Group B $55-64$ & $1.05(0.73-1.50)$ & 0.81 & $0.98(0.68-1.42)$ & 0.91 \\
\hline Group C $65-74$ & Reference & & Reference & \\
\hline Group D $\geq 75$ & $2.58(1.58-4.21)$ & 0.0002 & $2.84(1.70-4.74)$ & $<.0001$ \\
\hline Stage at $\mathrm{Dx}$ & & $<.0001$ & & $<.0001$ \\
\hline $1-2$ & Reference & & Reference & \\
\hline 3 & $0.94(0.61-1.46)$ & 0.79 & $0.92(0.58-1.47)$ & 0.72 \\
\hline 4 & $3.04(2.03-4.55)$ & $<.0001$ & $2.83(1.70-4.72)$ & $<.0001$ \\
\hline Gleason Score & & 0.0007 & & 0.0008 \\
\hline$<=6$ & Reference & & Reference & \\
\hline 7 & $1.31(0.65-2.61)$ & 0.45 & $1.41(0.68-2.93)$ & 0.36 \\
\hline $8-10$ & $2.34(1.22-4.50)$ & 0.01 & $2.57(1.28-5.17)$ & 0.008 \\
\hline $\mathrm{CCI}$ at Dx & & 0.71 & & 0.52 \\
\hline 0 & Reference & & Reference & \\
\hline $1-2$ & $0.95(0.68-1.32)$ & 0.75 & $0.91(0.64-1.31)$ & 0.62 \\
\hline $3+$ & $1.17(0.72-1.91)$ & 0.53 & $1.23(0.72-2.08)$ & 0.45 \\
\hline RT at Dx & & 0.001 & & 0.32 \\
\hline No & Reference & & Reference & \\
\hline Yes & $0.58(0.42-0.80)$ & & $0.82(0.56-1.21)$ & \\
\hline PSA at Diagnosis & & 0.003 & & 0.34 \\
\hline $0-<10$ & Reference & & Reference & \\
\hline $10-<20$ & $0.87(0.55-1.37)$ & 0.55 & $0.95(0.58-1.54)$ & 0.82 \\
\hline $20-<100$ & $0.83(0.56-1.24)$ & 0.37 & $0.71(0.46-1.08)$ & 0.10 \\
\hline$\geq 100$ & $1.84(1.17-2.89)$ & 0.008 & $0.75(0.45-1.26)$ & 0.28 \\
\hline Prostatectomy at Dx & & 0.08 & & 0.06 \\
\hline No & Reference & & Reference & \\
\hline Yes & $0.69(0.46-1.05)$ & & $0.65(0.41-1.02)$ & \\
\hline
\end{tabular}

Table 3. Median Time from Diagnosis to Development of CRPC.

\begin{tabular}{lllll}
\hline Age (y) & Group A <55 & Group B 55 - 64 & Group C 65 - 74 & Group D $\geq 75$ \\
\hline Med. Time (y) from Dx to CRPC (95\% CI)† & $2.1(1.5-2.9)$ & $2.8(1.8-3.7)$ & $3.4(2.7-4.5)$ & $1.3(0.9-1.9)$ \\
5-Year CRPC-free Survival (\%) (95\% CI) & $17(6-32)$ & $32(23-40)$ & $40(32-48)$ & $3(0.2-13)$
\end{tabular}

Abbreviations: Dx, diagnosis;y, years; CRPC, castration resistant prostate cancer; $\mathrm{m}$, months, († depicts log rank test statistical significance $\mathrm{p}<0.0001)$. 
Table 4. Univariable and Multivariable Cox-PH Regression Analysis of the Time from CRPC to Death.

\begin{tabular}{|c|c|c|c|c|}
\hline & Univariable & & Multivariable & \\
\hline & HR $(95 \% \mathrm{CI})$ & $p$-value & HR $(95 \%$ CI) & $p$-value \\
\hline Age at Diagnosis (years) & & 0.19 & & 0.19 \\
\hline Group $A<55$ & $0.96(0.61-1.52)$ & 0.85 & $0.73(0.44-1.20)$ & 0.21 \\
\hline Group B $55-64$ & $0.82(0.61-1.11)$ & 0.19 & $0.76(0.55-1.05)$ & 0.10 \\
\hline Group C $65-74$ & Reference & & Reference & \\
\hline Group D $\geq 75$ & $1.30(0.86-1.98)$ & 0.21 & $1.07(0.68-1.69)$ & 0.76 \\
\hline Presence of Visceral Disease (Yes vs No) & $1.95(1.41-2.70)$ & $<0.0001$ & $2.01(1.40-2.88)$ & $<0.0001$ \\
\hline Absolute PSA Nadir on ADT ( $\geq 4 \mathrm{vs}<4 \mathrm{ng} / \mathrm{ml}$ ) & $1.99(1.47-2.69)$ & $<0.0001$ & $1.90(1.37-2.64)$ & $<0.0001$ \\
\hline Time to Nadir on ADT ( $<6 \mathrm{vs} \geq 6$ months) & $2.27(1.71-3.01)$ & $<.0001$ & $1.64(1.19-2.26)$ & 0.003 \\
\hline Duration of Hormone Sensitivity ( $\geq 12 \mathrm{vs}<12 \mathrm{mo}$ ) & $0.61(0.42-0.88)$ & 0.009 & $0.66(0.46-0.94)$ & 0.02 \\
\hline PSA at Diagnosis & & 0.05 & & 0.01 \\
\hline $0-<10$ & Reference & & Reference & \\
\hline $10-<20$ & $0.85(0.55-1.32)$ & 0.46 & $0.81(0.49-1.33)$ & 0.40 \\
\hline $20-<100$ & $0.67(0.45-0.99)$ & 0.67 & $0.64(0.43-0.94)$ & 0.02 \\
\hline$\geq 100$ & $1.12(0.75-1.69)$ & 0.58 & $0.48(0.29-0.79)$ & 0.004 \\
\hline Gleason at Diagnosis & & 0.09 & & 0.63 \\
\hline$<=6$ & Reference & & Reference & \\
\hline 7 & $0.92(0.54-1.57)$ & 0.76 & $0.88(0.51-1.52)$ & 0.65 \\
\hline $8-10$ & $1.30(0.79-2.15)$ & 0.30 & $1.02(0.59-1.73)$ & 0.96 \\
\hline $\mathrm{CCI}$ at time of diagnosis & & 0.50 & & 0.22 \\
\hline 0 & Reference & & Reference & \\
\hline $1-2$ & $0.93(0.70-1.24)$ & 0.64 & $0.80(0.59-1.10)$ & 0.17 \\
\hline $3+$ & $1.20(0.79-1.82)$ & 0.39 & $0.81(0.51-1.26)$ & 0.35 \\
\hline Stage at Diagnosis & & $<0.0001$ & & 0.06 \\
\hline $1-2$ & Reference & & Reference & \\
\hline 3 & $0.75(0.51-1.11)$ & 0.15 & $1.01(0.68-1.50)$ & 0.20 \\
\hline 4 & $1.53(1.09-2.17)$ & 0.02 & $2.21(1.42-3.44)$ & 0.59 \\
\hline Time from Dx to CRPC (per 1 month increase) & $0.994(0.991-0.998)$ & 0.002 & $0.996(0.992-1.001)$ & 0.11 \\
\hline \multicolumn{5}{|l|}{ Peripheral Anti-androgen Withdrawal Response } \\
\hline No & Reference & & Reference & \\
\hline Yes & $0.89(0.65-1.23)$ & 0.47 & $0.85(0.58-1.23)$ & 0.39 \\
\hline
\end{tabular}

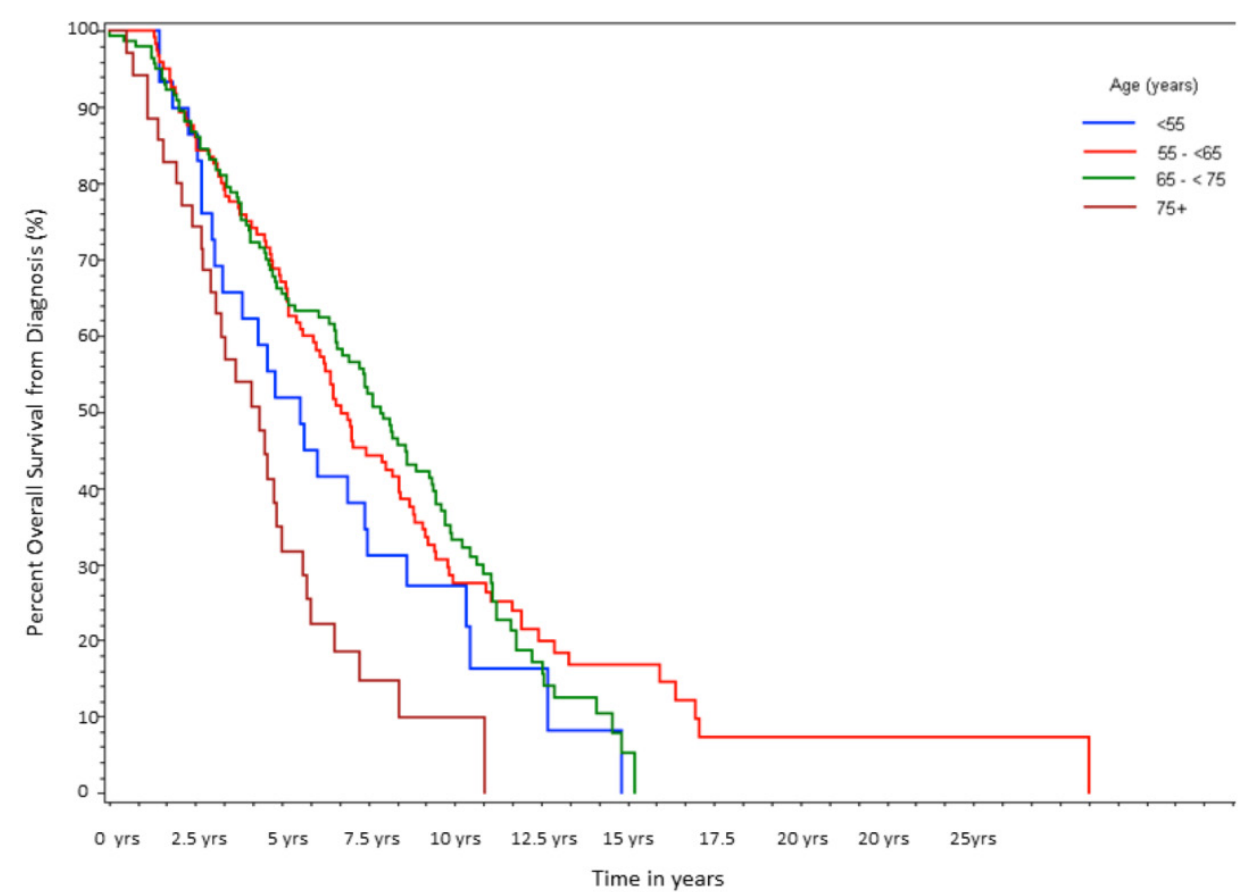

Figure I. Percent survival over time, stratified by age at initial diagnosis $(n=333)$. 


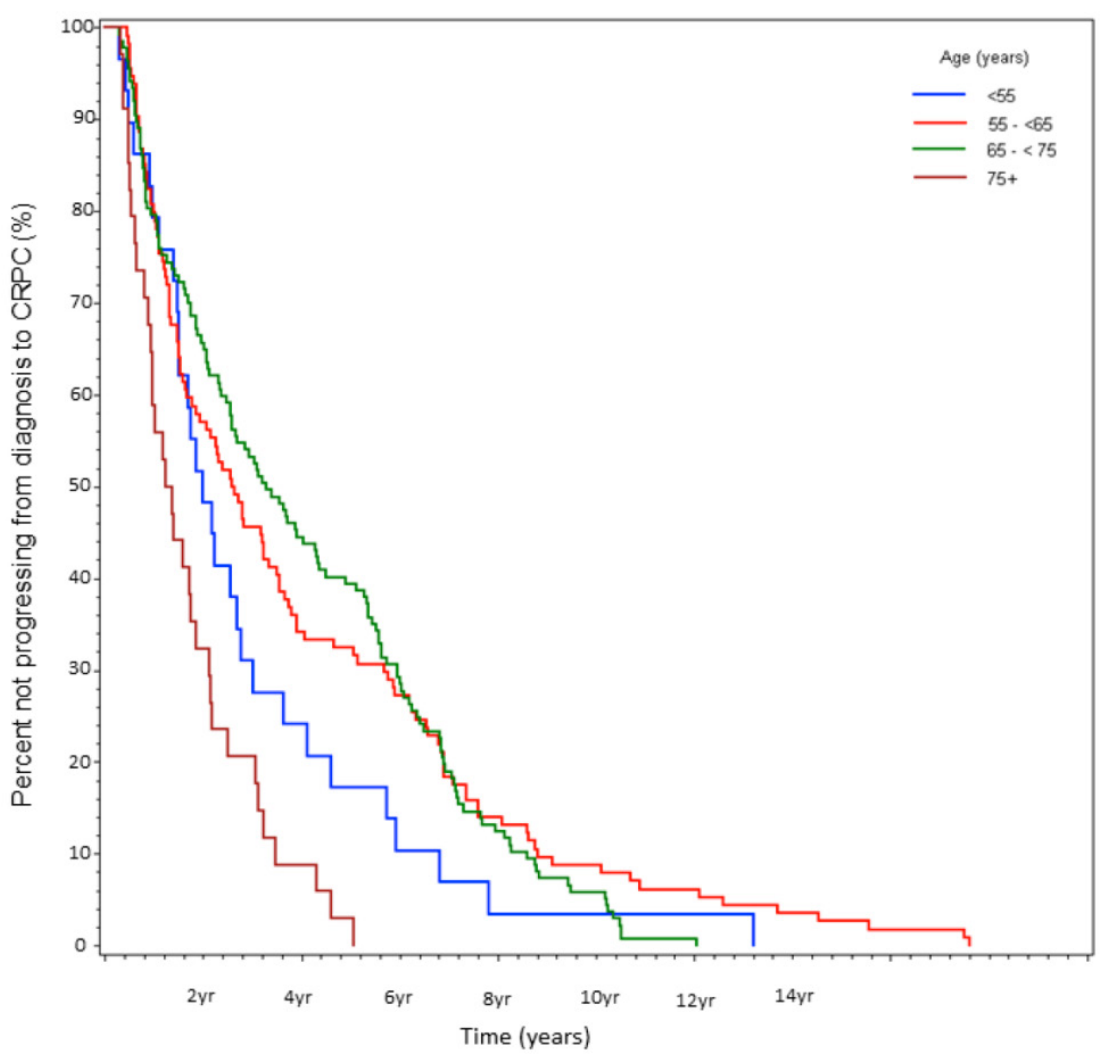

Figure 2. Percent not progressing from diagnosis to CRPC over time, stratified by age at initial diagnosis. $(n=3 \mid 4)$.

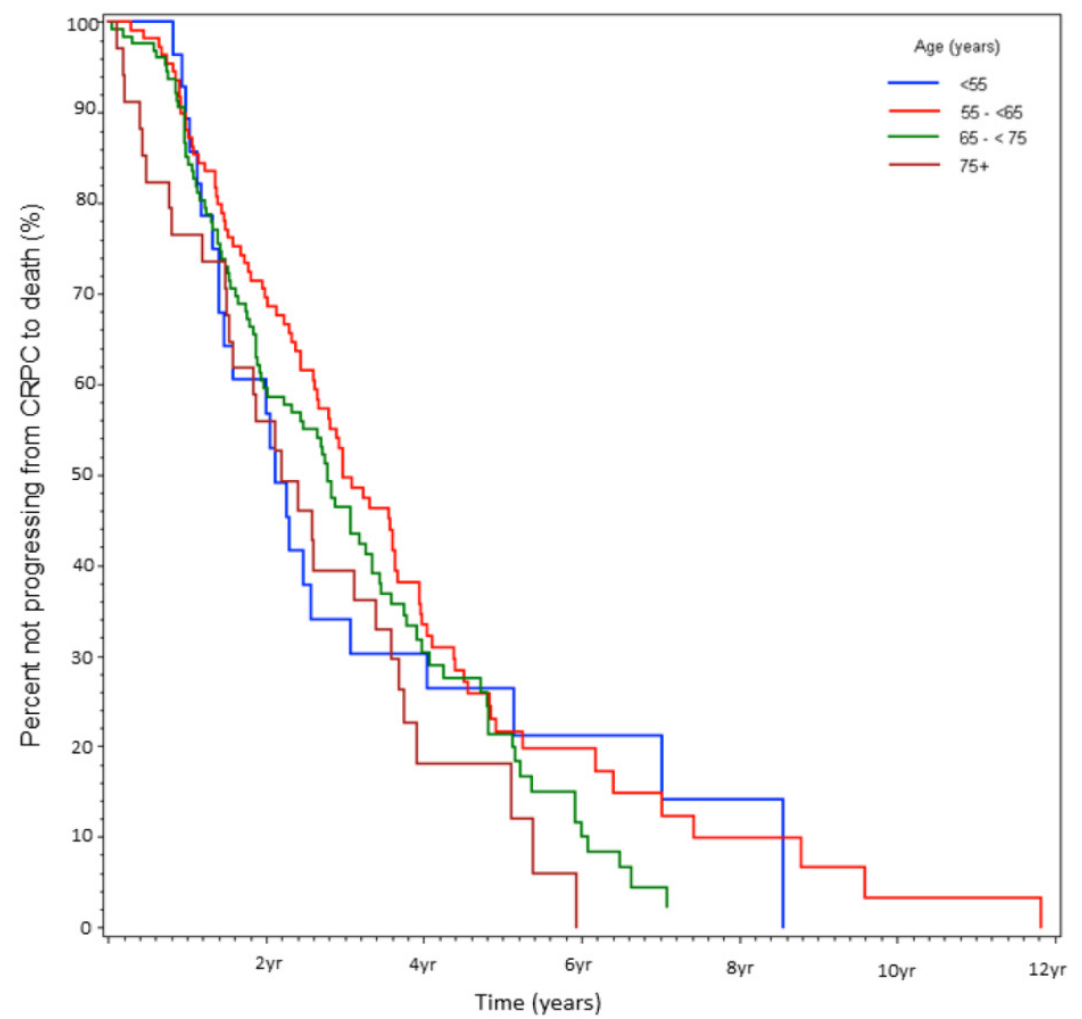

Figure 3. Percent not progressing from CRPC to death over time, stratified by age at initial diagnosis (\%). 


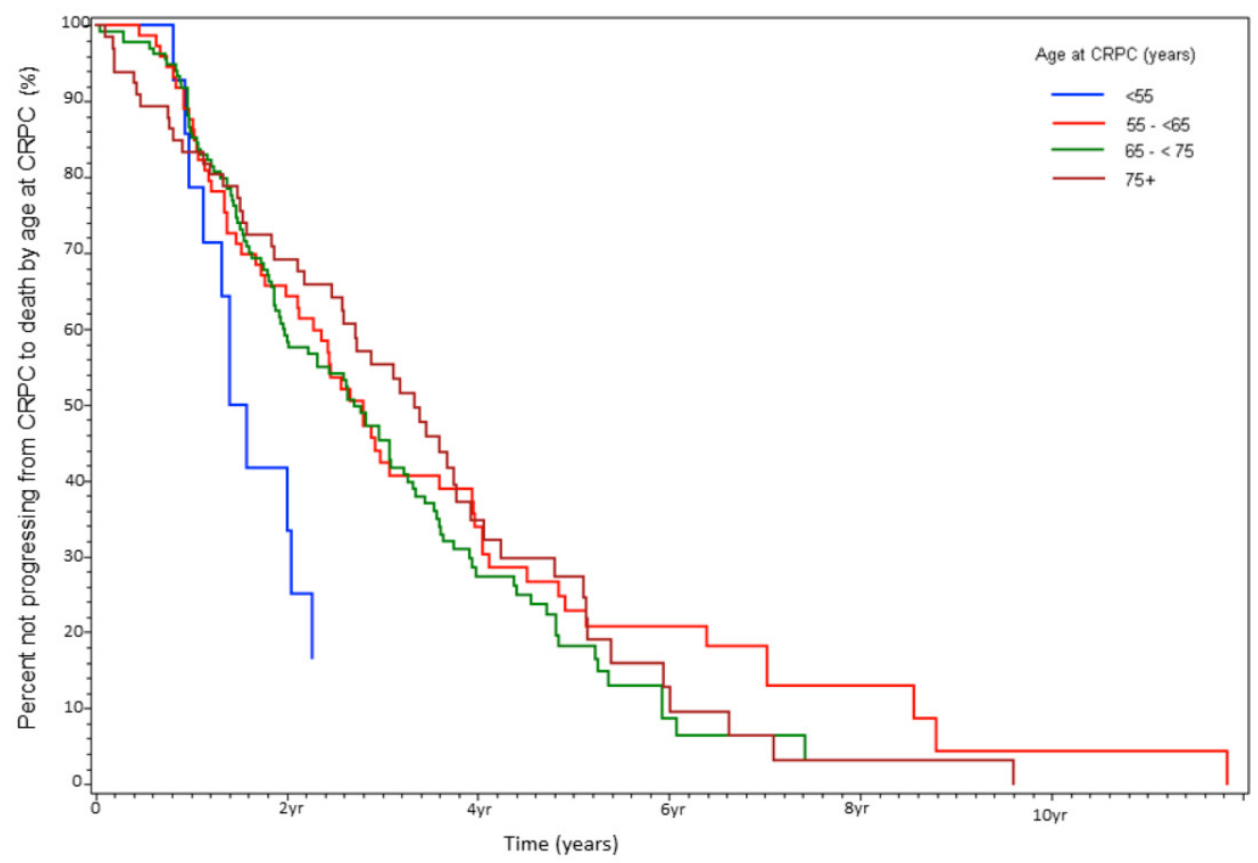

Figure 4. Percent not progressing from CRPC to death over time, stratified by age at CRPC diagnosis (\%).

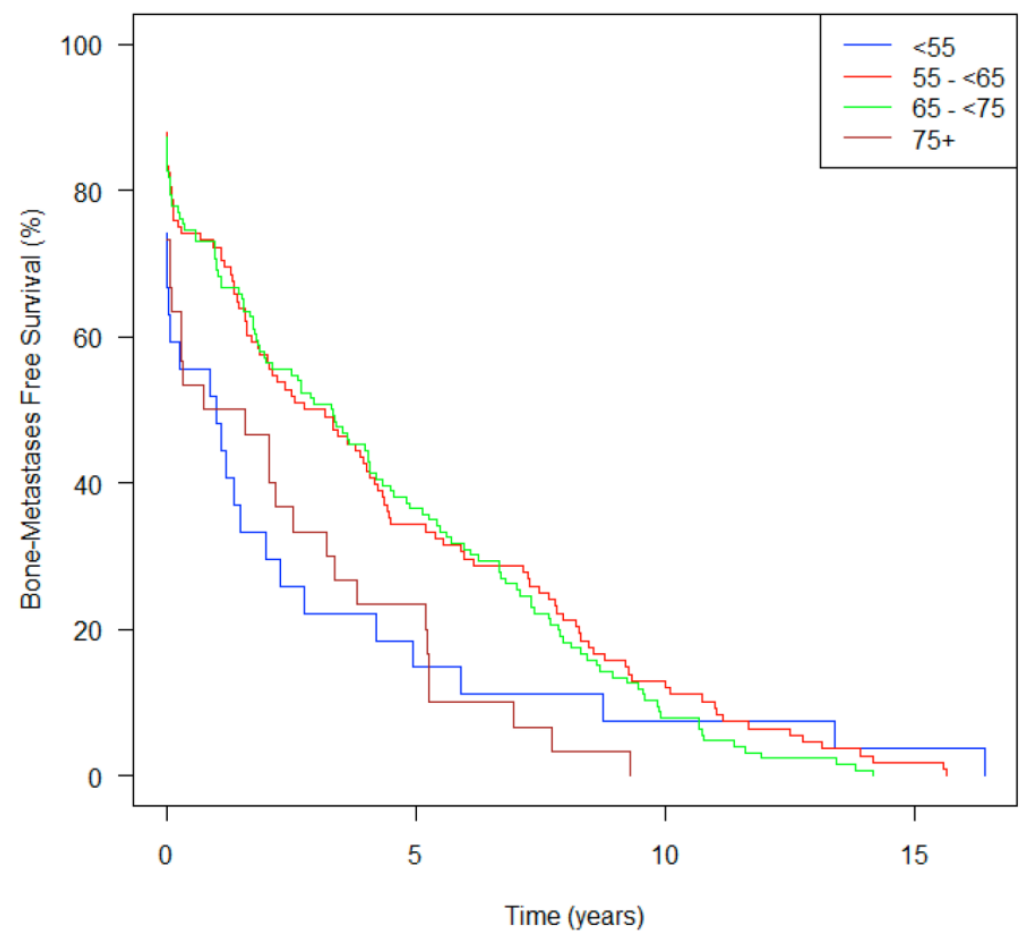

Figure 5. Bone metastasis-free survival from diagnosis over time, stratified by age at initial diagnosis $(n=333)$.

Time from Initial Diagnosis to Bone Metastasis

Patients in Group A, showed a median time from initial diagnosis to bone metastasis of only $1.2 \mathrm{yrs}$ (95\% CI 0.03-2.75) compared to 4.1 yrs (2.89-5.50) for Group C (Table 1). Figure 5 demonstrates that patients in Groups A and D both had an increased risk of developing bone metastases, and the curves continue to diverge for approximately ten years. Group A patients on univariate analysis had a HR of 1.55 , bordering on statistically significance $(p=0.067)$ and on multivariate analysis had a HR of $1.49(\mathrm{p}=0.13)$. 
Table 5. OS Multivariate Cox PH Regression of Significant Covariates in CRPC Patients Receiving Chemotherapy from Start of Chemotherapy.

\begin{tabular}{|c|c|c|c|c|}
\hline Variable & Reference & HR & $95 \% \mathrm{CI}$ & $p$ \\
\hline Age at Diagnosis (years) & & & & 0.58 \\
\hline Group A $<55$ & 65 to 74 & 1.18 & $0.70-2.01$ & 0.96 \\
\hline Group B 55 to 64 & 65 to 74 & 0.88 & $0.61-1.28$ & 0.50 \\
\hline Group D $\geq 75$ & 65 to 74 & 0.71 & $0.30-1.64$ & 0.42 \\
\hline ECOG & & & & 0.08 \\
\hline 2 & $0-1$ & 1.31 & $0.82-2.10$ & 0.25 \\
\hline$\geq 3$ & $0-1$ & 2.52 & $1.04-6.08$ & 0.03 \\
\hline PSA Doubling Time & & & & 0.003 \\
\hline$\geq 0$ and $<30$ days & $<0$ or $\geq 60$ & 1.86 & $1.21-2.86$ & 0.005 \\
\hline$\geq 30$ and $<60$ days & $<0$ or $\geq 60$ & 0.96 & $0.65-1.40$ & 0.64 \\
\hline $\log (\mathrm{ALP})$ & (cont.) & 1.02 & $0.85-1.24$ & 0.82 \\
\hline $\log (\mathrm{LDH})$ & (cont.) & 2.43 & $1.62-3.62$ & $<0.0001$ \\
\hline $\mathbf{H g b}(\mathrm{g} / \mathrm{L})$ & & & & 0.0002 \\
\hline$<100$ & $\geq 140$ & 3.62 & $1.77-7.42$ & 0.0004 \\
\hline $100-119$ & $\geq 140$ & 2.07 & $1.09-3.96$ & 0.03 \\
\hline $120-139$ & $\geq 140$ & 1.24 & $0.70-2.22$ & 0.46 \\
\hline \multicolumn{5}{|l|}{ Type of Chemotherapy } \\
\hline Docetaxel & Other & 0.47 & $0.32-0.69$ & 0.0001 \\
\hline
\end{tabular}

Abbreviations: OS, overall survival; CRPC, castration resistant prostate cancer; PSA, prostate specific antigen; ECOG, Eastern Cooperative Oncology Group; ALP, alkaline phosphatase; Hgb, hemoglobin.

\section{Chemotherapy Overall Survival Subset Analy- sis}

A subset analysis of 246 chemotherapy treated patients, with average follow-up of 1.1 yrs, observed 193 deaths. The median survival was 55 weeks (wks) with no differences on the basis of age stratification. Median PFS was 24.4 wks and median PSA-PFS was 26 wks, respectively, with no statistical difference between age groups. On multivariate analysis of significant covariates, PSA doubling time of less than 1 month, ECOG PS $\geq 3$, and baseline Hgb less than 100 $\mathrm{g} / \mathrm{L}$ were consistent predictors of shorter OS, PFS, and PSA-PFS. Elevated LDH was an important predictor of OS but not PFS or PSA-PFS. Treatment with docetaxel chemotherapy was statistically protective versus non-docetaxel based regimens for both OS and PFS on multivariate analysis ( $\mathrm{HR}=0.47, \mathrm{p}=0.0001$, and $\mathrm{HR}=0.64, \mathrm{p}=0.02$, respectively, Table 5).

\section{Discussion}

In this study of 333 CRPC patients, treated over a $10 \mathrm{yr}$ period, advanced age $(\geq 75)$ at the time of initial prostate cancer diagnosis is associated with a statistically significant shorter OS, from the time of initial diagnosis to death. Interestingly, we also show a trend towards worse survival in patients who were $\leq 55$ at the time of initial diagnosis. This is similar to the
SEER study showing a bimodal distribution of risk, based on age at diagnosis especially in patients with advanced stage or histology. ${ }^{14}$

Patients $\geq 75$ had a decreased OS (and prostate cancer specific OS) likely due to a shorter duration from diagnosis to CRPC. Our data suggests this is related to both advanced stage at diagnosis and a statistically significant shorter duration of hormone sensitivity. This latter finding is consistent with results presented by Hussain et al where a shorter duration of response to hormonal therapy correlated with a decreased OS. ${ }^{20}$ One reason for the reduced duration of hormone sensitivity may be lower pretreatment testosterone levels in the elderly population; though not directly measured in our study this has been linked to worse survival. ${ }^{21}$

Patients who were $<55$ at the time of diagnosis showed an unexpected trend towards worse survival (5.5 yrs versus 7.8 yrs) despite having fewer comorbidities and a better performance status. This group developed bone metastases earlier, a result that bordered on being statistically significant. At presentation a greater proportion of these patients had low PSA levels $(<10 \mathrm{ng} / \mathrm{ml})$, stage 4 disease, and visceral metastases. Further studies are needed to understand if there are unique host or tumor factors that lead to this presentation in younger patients or if these tumors are more poorly differentiated and non-PSA 
producing. A positive family history was also found in the majority of these patients, raising the question of whether there is a link between early onset prostate cancer, family history and genetics. Indeed such a link has been described. In one study up to $43 \%$ of patients under 55 had a genetic predisposition. ${ }^{22,23}$ While several mechanisms have been described, recent attention has focused on genes, such as the BRCA gene, which when mutated has been shown to confer an increased risk of recurrence following local therapy and increased prostate cancer-specific death (HR 5.16). ${ }^{24-26}$ Understanding mutations like BRCA may have important screening, diagnostic and therapeutic implications.

In terms of survival from CRPC to death, neither age at diagnosis, nor age at onset of CRPC impacted survival. This is consistent with an analysis of the TAX 327 study where age was not a statistically significant prognostic factor. ${ }^{27}$ Our results perhaps differ slightly from those presented by Halabi et al, which showed CRPC patients over 80 had worse outcomes than other age groups, but the age categories in these two studies were overlapping ( $\geq 75$ and $\geq 80$ ), there were relatively few patients in the advanced age groups in both studies, and the Halabi study predated the use of docetaxel chemotherapy. ${ }^{14}$ The key factors that were independently predictive of survival from CRPC to death were absolute PSA nadir on ADT $(<4$ vs $>4 \mathrm{ng} / \mathrm{ml}$ ), time to PSA nadir on ADT ( $<6 \mathrm{mo}$ vs $>$ $6 \mathrm{mos})$, duration of hormone sensitivity ( $<12$ vs $\geq 12$ mos), presence of bone metastases, and presence of visceral metastases (Table 4).

In patients receiving chemotherapy, consistent with other studies, a significantly reduced risk of death was seen for those who received docetaxel-based therapy. ${ }^{28,29} \mathrm{We}$ did not find that the mortality associated with chemotherapy was age-related, suggesting patients were appropriately selected and confirmed earlier observations that there is no strict age criteria that should preclude appropriate treatment. ${ }^{17}$ This is an important finding since even in our study we show that patients who were $\geq 75$, were less likely to receive chemotherapy than younger patients. Given both the palliative and survival benefits of chemotherapy, its use should therefore not be dictated by age alone.

\section{Conclusions}

In this study we evaluated CRPC patients over a $10 \mathrm{yr}$ period and showed that age at initial diagnosis of prostate cancer did influence outcomes with a bimodal survival curve. Poorer outcomes were most evident in the elderly $(>75)$ age group with an overall survival of only 4.3 yrs. In this group, the duration of hormone sensitivity was shorter, possibly owing to the reduced testosterone levels at baseline. Based on these results, it may be reasonable to prepare the very elderly patients that hormonal therapy may only have short term benefits and that chemotherapy may be needed to optimize disease control. A similar approach may also be warranted in younger patients where again outcomes appeared worse. One strategy to improve outcomes overall may be with the use of novel more effective hormonal treatments, such as abiraterone acetate (a CYP17 inhibitor) or enzalutamide (a new generation androgen receptor antagonist) earlier in the course of the disease to delay the development of CRPC; or alternatively introducing chemotherapy earlier in the course of treatment, especially in the younger and fitter patients. These are approaches currently under evaluation in clinical trials. Although age at initial diagnosis requires validation, it could be an important stratification factor for patients on clinical trials. As we look to the future, a more comprehensive understanding of prostate cancer from both a prognostic and molecular perspective may open further diagnostic and therapeutic avenues, allowing tailoring of therapy and ultimately lead to better outcomes in the future.

\section{Acknowledgements}

The authors would like to thank Cancer Care Ontario (CCO) for linking study patients with the CCO vital statistics registry, and Princess Margaret Hospital / University Health Network for providing biostatistics support and funding this study. The authors would also like to specifically thank Alisha Albert-Green, Manjula Maganti, and Melania Pintilie for their statistical support and expertise.

\section{Competing Interests}

The authors have declared that no competing interest exists.

\section{References}

1. Siegel R, Naishadham D, Jemel A, et al. Cancer statistics, 2012. CA Cancer J Clin 2012; 62: 10-29.

2. Kattan M, Cuzick J, Fisher G et al. A nomogram incorporating PSA level to predict cancer-specific survival for men with clinically localized prostate cancer managed without curative intent. Cancer 2007; 110: 69-74.

3. Shipley WU, Thames HD, Sandler HM et al. Radiation therapy for clinically localized prostate cancer: a multi-institutional pooled analysis. JAMA 1999; 281(17): 1598-1604.

4. Klotz K. Nomogram for predicting survival in men with clinically localized prostate cancer who do not undergo definitive therapy. Nat Clin Pract Urol 2008; 5(7): 362-363.

5. Tewari A, Gamito E, Crawford J et al. Biochemical recurrence and survival prediction models for the management of clinically localized prostate cancer. Clin Prostate Canc 2004; 2(4): 220-227.

6. Zagars G, von Eschenbach A, Ayala A. Prognostic factors in prostate cancer. Analysis of 874 patients treated with radiation therapy. Cancer 1993; 72: 1709-1725. 
7. Sandhu D, Munson K, Benghiat A et al. Natural history and prognosis of prostate carcdinoma in adolescents and men under 35 years of age. Br J Urol 1992; 69: 525-529.

8. Aprikian A, Zhang Z, Fair W. Prostate adenocarcinoma in men younger than 50 years. Cancer 1992; 74(6): 1768-1777.

9. Parker C, Gospodarowicz M, Warde P. Does age influence the behavior of localized prostate cancer? BJU Int 2001; 87: 629-637.

10. Abouassaly R, Paciorek A, Ryan C et al. Predictors of clinical metastasis in prostate cancer patients receiving androgen deprivation therapy. Cancer 2009; 115: 4470-4476.

11. Merrill RM, Bird JS. Effect of young age on prostate cancer survival: a population-based assessment (United States). Cancer Causes Control 2002; 13: 435-443.

12. Tward JD, Lee CM, Pappas LM, et al. Survival of men with clinically localized prostate cancer treated with prostatectomy, brachytherapy, or no definitive treatment: impact of age at diagnosis. Cancer 2006; 107: 2392-2400.

13. Halabi S, Small E, Kantoff P et al. Prognostic model for predicting survival in men with hormone-refractory metastatic prostate cancer. J Clin Oncol 2003; 21: 1232-1237.

14. Halabi S, Vogelzang N, Ou S et al. Clinical outcomes by age in men with hormone refractory prostate cancer: a pooled analysis of 8 cancer and leukemia group B (CALGB) studies. J of Urol 2006; 176: 81-86.

15. Armstrong AJ, Garett-Mayer ES, Yang YO et al. A contemporary prognostic nomogram for men with hormone-refractory metastatic prostate cancer: A TAX327 study analysis. Clin Cancer Res 2007;13(21): 6396-6403.

16. Therasse P, Arbuck S, Eisenhauer E et al. New guidelines to evaluated the response to treatment in solid tumors. European Organization for Research and Treatment of Cancer, National Cancer Institute of the United States, National Cancer Institute of Canada. J Natl Cancer Inst 2000; 92: 205-216.

17. Hall W, Jani A, Ryu J et al. The impact of age and comorbidity on survival outcomes and treatment patterns in prostate cancer. Prostate Cancer and Prostatic Diseases 2005; 8: 22-30.

18. Charlson $\mathrm{M}$, Pompei $\mathrm{P}$, Ales $\mathrm{K}$ et al. A new method of classifying prognostic comorbidity in longitudinal studies: development and validation. J Chron Dis 1987; 40: 373-383.

19. Scher HI, Halabi S, Tannock I et al. Design and end points of clinical trials for patients with progressive prostate cancer and castrate levels of testosterone: recommendations of the prostate cancer clinical trials working group. J Clin Oncol 2008; 26: 1148-1159.

20. Hussain $\mathrm{MH}$, Goldman $\mathrm{B}$, Tangen $\mathrm{CM}$, et al. Use of prostate-specific antigen progression (PSA-P) to predict overall survival (OS) in patients (pts) with metastatic prostate cancer (PC): Data from S9346 and S9916. J Clin Oncol. 2009; 27(15): 2450-2456.

21. Chodak G, Vogelzang N, Caplan R et al. Independent prognostic factors in patients with metastatic (stage D2) prostate cancer. The Zoladex study group. JAMA 1991; 265(5): 618-621.

22. Steinberg G, Carter B, Beaty T et al. Family history and the risk of prostate cancer. Prostate 1990; 17: 337-347.

23. Carter B, Beaty T, Steinberg G et al. Mendelian inheritance of familial prostate cancer. Proc Natl Acad Sci 1992; 89: 3367-3371.

24. Forrest M, Edwards S, Houlston R et al. Association between hormonal genetic polymorphisms and early-onset prostate cancer. Prostate Cancer and Prostatic Diseases 2005; 8: 95-102.

25. $\mathrm{Xu} \mathrm{J}$, Zheng SL, Isaacs SD et al. Inherited genetic variant predisposes to aggressive but not indolent prostate cancer. PNAS 2010; 107(5): 2136-2140.

26. Gallagher, DJ, Gaudet MM, Pal P et al. Germline BRCA mutations denote a clinicopathologic subset of prostate cancer. Clin Cancer Res 2010; 16(7): 2115-2121.

27. Armstrong AJ, Garret-Mayer ES, Yang YO, et al. A contemporary prognostic nomogram for men with hormone-refractory metastatic prostate cancer: a TAX327 study analysis. Clin Cancer Res 2007; 13: 6396-6403.

28. Tannock IF, DeWit R, Berry W et al. Docetaxel plus prednisone or mitoxantrone plus prednisone for advanced prostate cancer. N Engl J Med 2004; 351:1502-1512.

29. Petrylak D, Tangen C, Hussain M et al: Docetaxel and estramustine compared with mitoxantrone and prednisone for advanced refractory prostate cancer. N Engl J Med 2004; 351: 1513-1520. 